\title{
MÉTODOS DE EVALUACIÓN PARA MOVILIZACIÓN MANUAL DE PACIENTES
}

\author{
EVALUATION METHODS OF PATIENT MANUAL HANDLING
}

\section{Juan Pablo Cortés Hoyos ${ }^{1}$}

\begin{abstract}
Resumen: El presente artículo parte del principio básico de la ergonomía, que se basa en la adaptación del trabajo a las capacidades de los individuos, disciplina que contempla, entre otros campos de acción, la movilización de pacientes hospitalizados o con atención domiciliaria, actividad que implica riesgos para el profesional de la salud o cuidador. En este campo de la ergonomía, se encuentran en la literatura un número considerable de artículos sobre metodologías de evaluación de los riesgos asociados a la movilización de pacientes. En este documento se hace una descripción de los métodos de evaluación de riesgos, clasificados en métodos ergonómicos para la evaluación de tareas, métodos multifactoriales de gestión de riesgos y métodos biomecánicos. A partir de este ejercicio descriptivo, se realiza un análisis de diferentes métodos de evaluación de riesgos, que contribuye a aportar a la construcción de modelos de movilización de pacientes con una mirada integral que minimice los riesgos.
\end{abstract}

Palabras clave: Métodos de evaluación de riesgo, movilización de pacientes.

\begin{abstract}
This article is based on the basic principle of ergonomics, which is based on the adaptation of work to the capacities of individuals, a discipline that contemplates, among other fields of action, the mobilization of hospitalized patients or with home care, an activity that involves risks for the healthcare professional or caregiver. In this field of ergonomics, a considerable number of articles are found in the literature on methodologies for assessing the risks associated with the mobilization of patients. This document provides a description of the risk assessment methods, classified into ergonomic methods for task assessment, multifactorial risk management methods and biomechanical methods. Based on this descriptive exercise, an analysis of different risk assessment methods is carried out, which contributes to the construction of patient mobilization models with a comprehensive view that minimizes risks.
\end{abstract}

Keywords: Risk assessment methods, patient mobilization.

\footnotetext{
${ }^{1}$ Investigador independiente. Manizales, Colombia. Correo electrónico: juanpablocortes3002@gmail.com. Orcid: https://orcid.org/oooo-0oo1-5839-4178
} 


\section{Introducción}

Del principio básico de la ergonomía consiste en adaptar el trabajo a las capacidades y posibilidades del individuo. Para el caso de la movilización o transferencia de pacientes hospitalizados o con atención domiciliaria, esta actividad laboral implica riesgos que deben controlarse con medidas que aporten a su reducción en un entorno especial debido a los movimientos imprevistos, grandes esfuerzos y posturas forzadas (Alba, 2016).

La importancia de realizar una revisión de los métodos que permiten evaluar los riesgos presentes en la movilidad de pacientes, según Salazar-Montes et al. (2016), se debe al aumento considerable de adultos mayores que requieren de un cuidador o acompañante que los apoye en la realización de las actividades de la vida diaria, que generalmente, corresponde a un miembro de la familia o cuidador informal que no posee los conocimientos adecuados para prevenir los riesgos, y por consiguiente, las enfermedades musculoesqueléticas. Es posible afirmar a partir de los datos de ausentismo en los hospitales e instituciones prestadores de servicios de salud, que la población mayormente afectada son mujeres, que padecen lesiones musculoesqueléticas debido a sobreesfuerzos realizados durante la manipulación de pacientes en los centros hospitalarios y el hogar.

El objetivo de este estudio es revisar los diferentes métodos que evalúan los determinantes ergonómicos en la movilización manual de pacientes en los centros hospitalarios y el hogar, con el fin de identificar los aspectos generadores de riesgos de lesiones de los trabajadores y cuidadores.

\section{Materiales y métodos}

Mediante una búsqueda realizada entre los años 2012 a 2018, se estableció la existencia de trece artículos publicados que recogen investigaciones en donde se aplican los métodos para la evaluación de riesgos por movilización de pacientes. Se clasificaron en los siguientes grupos definidos por Díaz et al. (2017) y Salazar-Montes et al. (2016):

1. Métodos ergonómicos para la evaluación de tareas.

2. Métodos multifactoriales de gestión de riesgos.

3. Métodos biomecánicos.

Una vez seleccionadas las fuentes de información de las bases de datos, catálogos online y motores de búsqueda, se hace la selección de artículos que permiten recoger los métodos de evaluación de riesgos para la movilización de pacientes. En la tabla 1 se especifican las fuentes analizadas. 
Tabla 1. Artículos sobre metodologías de evaluación de riesgos en la movilización de pacientes.

\begin{tabular}{|c|c|}
\hline Título del artículo & Autores y año de publicación \\
\hline $\begin{array}{l}\text { Aplicación del método "MAPO” para } \\
\text { evaluar el riesgo biomecánico por } \\
\text { movilización de pacientes en auxiliares de } \\
\text { enfermería del hospital General San Isidro } \\
\text { de la ciudad de Manizales. }\end{array}$ & Bernal-Osorio et al. (2018). \\
\hline $\begin{array}{l}\text { Nivel de riesgo biomecánico por } \\
\text { manipulación manual de pacientes adultos } \\
\text { en el área de hospitalización de una } \\
\text { institución de salud de alta complejidad en } \\
\text { Soledad, 2016-1. }\end{array}$ & $\begin{array}{l}\text { Puello-Mercado \& Medina } \\
\text { (2017). }\end{array}$ \\
\hline $\begin{array}{l}\text { Papel de la ergonomía hospitalaria frente } \\
\text { al manejo del paciente adulto por personal } \\
\text { de auxiliar de enfermería. }\end{array}$ & Díaz et al. (2017). \\
\hline $\begin{array}{l}\text { Ergonomía del trabajo de enfermeras en el } \\
\text { manejo manual de pacientes con } \\
\text { metodología REBA y MAPO. }\end{array}$ & Morales-Perrazo et al. (2017). \\
\hline $\begin{array}{l}\text { Ergonomía aplicada a la movilización de } \\
\text { pacientes en un servicio de hospitalización } \\
\text { mediante el método MAPO. }\end{array}$ & Alba (2016). \\
\hline $\begin{array}{l}\text { Equipo biomecánico para la movilización } \\
\text { de pacientes sin autonomía en el personal } \\
\text { de enfermería del hospital Andino } \\
\text { Alternativo de Chimborazo }\end{array}$ & Uvida (2016). \\
\hline $\begin{array}{l}\text { Intervenciones de enfermería para } \\
\text { disminuir la sobrecarga en cuidadores: Un } \\
\text { estudio piloto. }\end{array}$ & Sánchez et al. (2016). \\
\hline $\begin{array}{l}\text { Evaluación e intervención de la sobrecarga } \\
\text { del cuidador informal de adultos mayores } \\
\text { dependientes: Revisión de artículos } \\
\text { publicados entre } 1997-2014 .\end{array}$ & Salazar-Montes et al. (2016). \\
\hline $\begin{array}{l}\text { El papel de la ergonomía en la prevención } \\
\text { y evaluación de la carga física en un centro } \\
\text { hospitalario: Evaluación de las tareas de } \\
\text { movilización de pacientes mediante el } \\
\text { método MAPO Index. }\end{array}$ & Robla-Santos (2015). \\
\hline $\begin{array}{l}\text { Riesgo ergonómico asociado a } \\
\text { sintomatología musculoesquelética en } \\
\text { personal de enfermería. }\end{array}$ & Montalvo-Prieto et al. (2015). \\
\hline $\begin{array}{l}\text { Prevención de riesgos laborales en la } \\
\text { movilización de pacientes en una planta de } \\
\text { hospitalización. }\end{array}$ & Alcazar-Belchí (2015). \\
\hline $\begin{array}{l}\text { Association of job strain with working } \\
\text { hours, shift-dependent perceived } \\
\text { workload, sleepiness and recovery. }\end{array}$ & Karhula et al. (2013). \\
\hline $\begin{array}{l}\text { Flying through the hospital: Efficiency and } \\
\text { safety of an ergonomic solution. }\end{array}$ & Knibbe et al. (2012). \\
\hline
\end{tabular}




\section{Resultados y discusión}

De acuerdo con la metodología empleada y mediante el uso del Atlas ti como herramienta tecnológica que se observa en la figura 1, se reconoce que se requiere de un análisis multifactorial de riesgo de la movilización manual de paciente, abordado desde metodologías cualitativas y cuantitativas a partir de estudios transversales y descriptivos. En los artículos revisados se han analizado los riesgos biomecánicos por la manipulación manual de pacientes en el personal de enfermería (Montalvo-Prieto et al., 2015, Alcazar-Belchí, 2015). También se encontraron artículos que analizan los riesgos posturales en la movilización de pacientes en donde el cuidador informal presenta riesgos laborales, asociados no solo a trastornos musculoesqueléticos, sino también emocionales y psiquiátricos debido a la multiplicidad de factores que intervienen en esta actividad (Salazar-Montes et al. 2016).

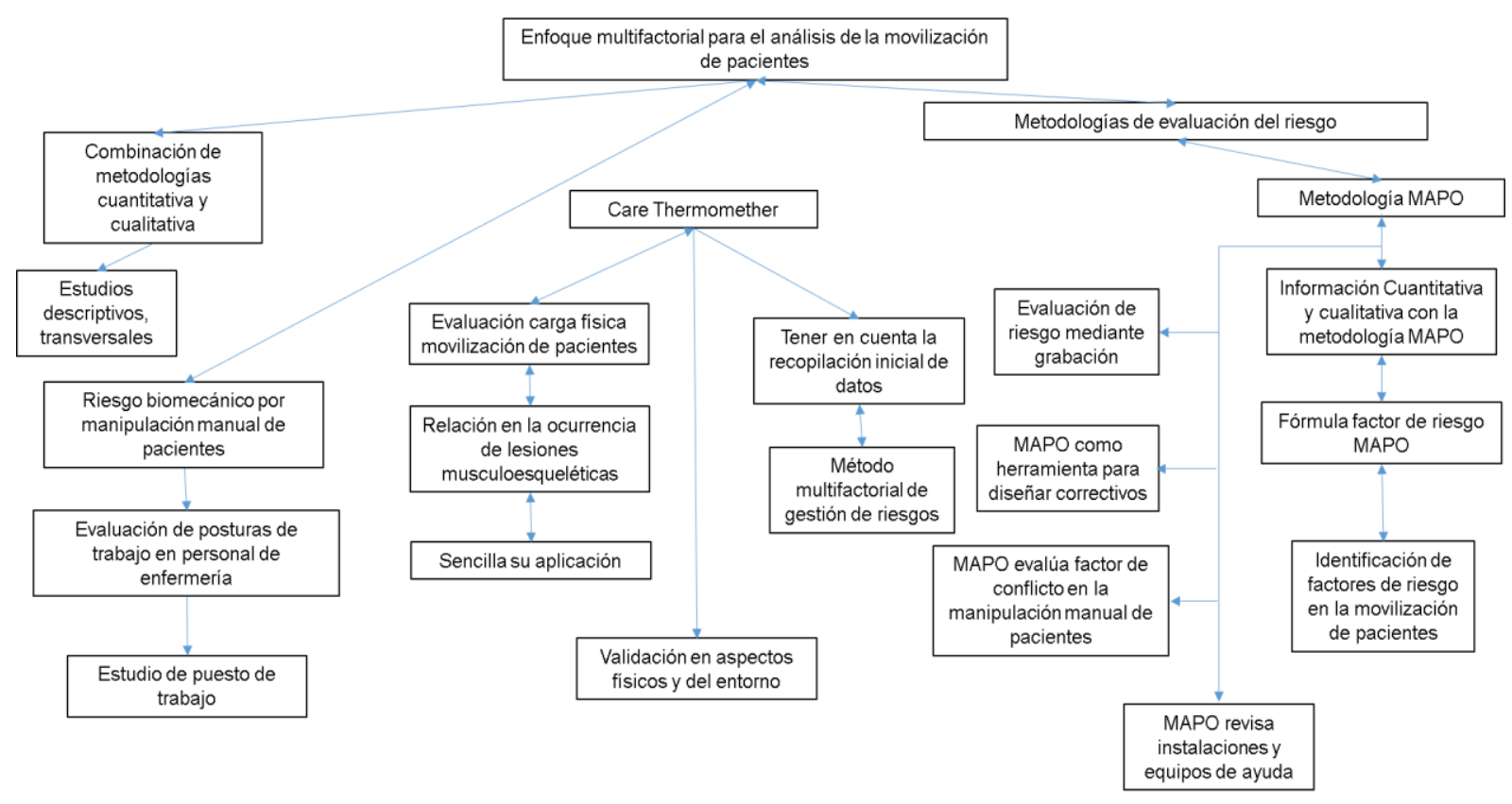

Figura 1. Red Semántica métodos de evaluación de riesgos de movilización de pacientes. Fuente: elaboración propia.

En la búsqueda realizada también se encontró que el método MAPO es la más divulgada en la evaluación de riesgos por la movilidad de pacientes, ya que recoge diferentes variables en la evaluación de riesgos como son: estudio del puesto de trabajo y el índice de exposición al riesgo y el uso de metodologías cuantitativas y cualitativas (Bernal-Osorio et al., 2018, Morales-Perrazo et al., 2017, Alba, 2016).

El nivel de colaboración de los pacientes, el uso de silla de ruedas, las ayudas mecánicas, el lugar de movilización y la infraestructura, se consideran variables importantes a tener en cuenta en la selección metodológica para el análisis biomecánico de la manipulación manual de pacientes que ocurren dentro de un centro hospitalario y en un hogar (Bernal-Osorio et al., 2018). 
En cuanto a las condiciones específicas que contribuyen a la calificación y ponderado de los diferentes métodos analizados, es importante que se realice un análisis ergonómico de la actividad y las variables, considerando los cargos que se tienen dentro de una institución o en el hogar, ya que de esto dependen las condiciones que determinarán la evaluación de los factores riesgo.

Las tareas de empuje y arrastre de pacientes implican de un nivel de esfuerzo mayor del que se requiere en otras actividades. Se resalta la importancia que se debe tener con respecto a las características individuales, físicas y antropométricas de los trabajadores que pueden contribuir a generar alteraciones musculoesqueléticas en la población que requiere la movilización manual de pacientes (Morales-Perrazo et al., 2017).

En la tabla 2 se presentan los métodos para la evaluación de riesgos de la manipulación manual de pacientes, los autores, la clasificación de los aspectos ergonómicos que consideran, y la descripción de cada uno de acuerdo a lo identificado en la revisión de los diferentes artículos.

Tabla 2. Descripción de métodos de evaluación de riesgos de movilización de pacientes.

\begin{tabular}{|l|l|l|l|}
\hline \multicolumn{1}{|c|}{ Metodología } & \multicolumn{1}{|c|}{ Autores } & \multicolumn{1}{c|}{ Clasificación } & \multicolumn{1}{c|}{ Descripción } \\
\hline $\begin{array}{l}\text { Dortmund } \\
\text { Approach }\end{array}$ & Jäger et al. (2010) & $\begin{array}{l}\text { Método ergonómico de } \\
\text { evaluación de tareas }\end{array}$ & $\begin{array}{l}\text { Evalúa la sobrecarga } \\
\text { biomecánica lumbar } \\
\text { derivada del manejo } \\
\text { manual de los pacientes, } \\
\text { determinando el peso } \\
\text { soportado y, la capacidad } \\
\text { de carga de la columna } \\
\text { vertebral en el manejo } \\
\text { manual de pacientes. }\end{array}$ \\
\hline $\begin{array}{l}\text { Care } \\
\text { Thermometer }\end{array}$ & $\begin{array}{l}\text { Knibbe \& Friele } \\
\text { (1999) }\end{array}$ & $\begin{array}{l}\text { Método multifactorial } \\
\text { de gestión de riesgos }\end{array}$ & $\begin{array}{l}\text { Herramienta para evaluar } \\
\text { la carga física durante el } \\
\text { manejo de pacientes; } \\
\text { tiene en cuenta el uso de } \\
\text { equipos de ayuda y la } \\
\text { carga asistencial. }\end{array}$ \\
\hline $\begin{array}{l}\text { PTAI (Patient } \\
\text { Transfer } \\
\text { Assessment } \\
\text { Instrument) }\end{array}$ & Karhula et al. (2009) & Método biomecánico & $\begin{array}{l}\text { Herramienta que permite } \\
\text { evaluar la sobrecarga } \\
\text { biomecánica soportada } \\
\text { durante el traslado de } \\
\text { pacientes. }\end{array}$ \\
\hline $\begin{array}{l}\text { MAPO (Método } \\
\text { Movement and } \\
\text { Assistance } \\
\text { Hospital } \\
\text { Patients) }\end{array}$ & Battevi et al. (2006) & $\begin{array}{l}\text { Método multifactorial } \\
\text { de gestión de riesgos }\end{array}$ & $\begin{array}{l}\text { Movimiento y asistencia } \\
\text { de los pacientes; permite } \\
\text { evaluar el nivel de } \\
\text { exposición al riesgo por } \\
\text { manipulación manual de } \\
\text { pacientes. }\end{array}$ \\
\hline
\end{tabular}


Principalmente, es el personal de enfermería quien se expone a los esfuerzos físicos durante la movilización de pacientes. Para evaluar el nivel de esfuerzo se han diseñado diferentes métodos que analizan las condiciones que pueden generar riesgos y lesiones musculoesqueléticas. Entre los métodos de evaluación de riesgo, los más utilizados corresponden al MAPO y CARE TERMOMETHER.

El método MAPO (manejo y asistencia de pacientes hospitalizados) constituye una herramienta fundamental para diseñar programas que incorporen acciones correctivas necesarias. La finalidad es asegurar la salud ocupacional, evitar accidentes y enfermedades profesionales a largo plazo asociadas al manejo manual de pacientes (Tamminen-Peter et al., 2008). Evalúa criterios donde se especifican las causas de los trastornos musculoesqueléticos tales como, la carga asistencial dada por la presencia de pacientes dependientes, el tipo y grado de discapacidad motora de los pacientes, las características estructurales del ambiente de trabajo en el centro sanitario, los equipos de trabajo y su adecuación a la tarea, y la formación e información de los trabajadores sobre técnicas de movilización de pacientes. Permite realizar la identificación de diferentes variables para determinar el nivel de riesgo biomecánico al que está expuesto el personal de enfermería, de acuerdo a las características que presenta cada área de atención. El análisis de los resultados contribuye a tomar acciones correctivas y preventivas para evitar la presencia de accidentes o enfermedad laboral (BernalOsorio et al., 2018).

El método Care Thermomether se encuentra validado en Estados Unidos, Países Bajos, Alemania y Reino Unido, a partir del análisis de la aplicación en la atención de 1808 pacientes. De acuerdo a estos estudios de validación, existe una correlación entre altos niveles de exposición a cargas físicas y los trastornos musculoesqueléticos. Por lo tanto, la evaluación de la exposición es un medio sensible y práctico para diseñar y evaluar los factores de riesgo relacionados con la movilización manual de pacientes. En la práctica, durante la aplicación de este método, de debe prestar especial atención a la fase de recopilación de datos, teniendo en cuenta que debe ser cuidadosa, puntual y rigurosa, ya que esto juega un papel crucial en la obtención de resultados precisos y útiles en la evaluación de los trastornos musculoesqueléticos (Knibbe et al., 2012).

De acuerdo con lo anterior, es necesario el entrenamiento del personal encargado de aplicar los diferentes métodos, enfocándose en la forma de recopilar la información, y lo que se va a evaluar específicamente dentro de cada ítem, para evitar errores en los resultados y conclusiones.

Según Knibbe \& Knibbe (2012), el uso de la eslinga con arnés disminuye el esfuerzo en los trabajadores encargados de hacer los traslados de los pacientes cuando se requiere hacer alguna movilización manual.

En las actividades de movilización de pacientes, los accidentes laborales de origen musculoesquelético representan una elevada tasa de incidencia, generalmente debido a condiciones de trabajo inadecuadas. Por esto es que resulta importante, considerar variables adicionales, como la edad, antigüedad en el puesto de trabajo y el estado de salud de los trabajadores. La metodología MAPO contribuye a analizar los aspectos propios del trabajo, pero no es suficiente en la identificación de las condiciones propias del trabajador, es decir 
aspectos individuales de las personas que se encargan de hacer la movilización manual de pacientes (Battevi et al., 2006).

En el análisis de las lesiones asociadas a la movilización manual de pacientes, se hace importante analizar en base a criterios epidemiológicos, la información que deriva de la vigilancia de la salud de los trabajadores, y que debe suponer la aplicación de protocolos y guías clínicas que tengan en cuenta y permitan identificar la posible incidencia de los factores de riesgo de origen laboral en el estado de salud del trabajador (Malo et al., 2012, NoriegaElío et al., 2005).

Según el análisis de las condiciones individuales, actúa como factor potenciador del riesgo de carga física, la movilización de pacientes obesos parcialmente colaboradores y no colaboradores, con uso de rodillo y sábanas, así como la dificultad para la aplicación de los estándares posturales en esta tipología de pacientes (Álvarez-Casado et al., 2010).

A pesar de la estandarización de medidas, pesos, guías y manuales para el manejo de pacientes, los trastornos musculoesqueléticos en el personal de salud continúan apareciendo, ligados a condiciones locativas inapropiadas, personal insuficiente para las demandas de las tareas y asignación de recursos insuficientes para invertir en equipos e infraestructura (Díaz et al., 2017).

En cuanto a los aspectos adicionales, la duración y organización de la jornada laboral representa una importante variable en la identificación de factores que implican sobreesfuerzos en el personal encargado de hacer la movilización manual de pacientes, ya que se incrementa el tiempo de exposición y las condiciones físicas identificadas en las metodologías de análisis biomecánico. Excederse en el número de horas que se laboran en una semana constituye, de por sí, un factor de riesgo para el trabajador, porque los músculos, tendones, cápsulas y ligamentos que forman la estructura del cuerpo humano y que permiten el movimiento y la realización de diversas actividades, requieren de reposo para su recuperación, de lo contrario se genera fatiga (Montalvo-Prieto et al., 2015).

Como se identifica dentro de algunas de las investigaciones, los trastornos musculoesqueléticos que refieren el personal de enfermería se enfocan específicamente en la zona dorso-lumbar. De acuerdo a Montalvo-Prieto et al. (2015), auxiliares de enfermería con una carga laboral elevada, que llevan más de tres años en una institución de salud, refieren que al menos han generado alguna molestia musculoesquelética en la población femenina.

El control de los factores de riesgo en la movilización manual de pacientes involucra a la organización en su conjunto, requiriendo del compromiso tanto de los directivos como de sus trabajadores según Robla-Santos et al., (2010).

Son factores relevantes a considerar, no solo aquellos que involucran directamente al personal de salud que realiza la movilización de pacientes, sino también otros aspectos que provienen desde la gestión de los directivos, en cuanto al mantenimiento de las condiciones óptimas de trabajo y de la infraestructura física, y en relación a aspectos organizacionales.

Los factores que consideran los métodos analizados, especialmente MAPO, no son suficientes para identificar las condiciones que ponen en riesgo al sistema musculoesquelético, por lo que se amplían en una investigación donde se incorporaron otros 
métodos de evaluación de carácter cuantitativo (software ergo-cargas) y cualitativo (estudio del puesto de trabajo), comprobándose la relevancia de estrategias integrativas, para observar y conocer con mayor información la realidad en cuanto a los riesgos del manejo manual (Álvarez-Casado et al., 2010, Battevi et al., 2006).

Dentro de las variables que coincide en todos los métodos, se encuentra el peso soportado por el equipo al realizar la movilización manual de pacientes. De acuerdo a un estudio realizado por Jorgensen et al. (1999), modificaciones en el peso de levantamiento, implica cambios en la respuesta fisiológica del organismo del trabajador, y con ello, del riesgo de lumbalgia. Esto comprueba que este tipo de actividad representa un factor de riesgo importante para la presencia de dolor lumbar.

En concordancia con lo anterior, el peso máximo recomendado de acuerdo a las diferentes metodologías de análisis, disminuye gradualmente conforme a la reducción de la altura de levantamiento inicial, y cuando el objeto se encuentra por arriba del hombro del trabajador, el peso máximo recomendado disminuye drásticamente. Esta situación comprueba que, al modificar alguna de las variables en relación a las condiciones ergonómicas de la actividad, es posible disminuir el riesgo para la salud. El peso en kilogramos, determina de manera importante el riesgo vinculado a los trastornos musculoesqueléticos. Existen peligros potenciales asociados al levantamiento manual de cargas superiores a diez kilogramos y al inadecuado procedimiento de movilización realizado por los trabajadores (movimientos de empuje/arrastre), y además, a un insuficiente entrenamiento en el uso de equipos (Chen \& Yang, 2006).

\section{Conclusiones}

La preparación y conocimiento previo en los métodos de evaluación de los riesgos asociados a la manipulación manual de pacientes, es importante para evitar la ocurrencia de errores en su aplicación, que generen inadecuadas interpretaciones de los resultados y conclusiones. Si bien los principales métodos de evaluación son el MAPO y CARE TERMOMETHER, es necesario que en la evaluación se consideren variables adicionales para realizar una estimación más precisa del riesgo.

El uso de grúas o eslingas para la movilización de los pacientes es un recurso muy valioso para disminuir los esfuerzos físicos para los miembros superiores y columna vertebral, a pesar que inicialmente se debe entrenar al personal para lograr de manera efectiva estos traslados. Es el método más efectivo que involucra a una sola persona y permite generar el mínimo esfuerzo y facilitar los traslados de los pacientes. En Latinoamérica y Colombia esta tecnología representa costos importantes dentro del sector salud, pero que debería ser la mejor opción para evitar la generación de accidentes laborales y evitar trastornos musculoesqueléticos. Por lo tanto, el uso de ayudas mecánicas contribuye a la disminución del esfuerzo en el momento de hacer cualquier tipo de traslado, mitigando e incluso eliminando el riesgo.

Aun cuando, de acuerdo a los estudios, el personal de la salud encargado de realizar la movilización manual de pacientes percibe tener conocimiento y formación sobre la forma 
correcta de realizar las manipulaciones, se observa que las condiciones del trabajo determinan la adopción de posturas, generación de movimientos inadecuados y sobre esfuerzos, que incrementan el riesgo de sufrir de algún trastorno musculoesquelético.

\section{Referencias}

Alba, R. (2016). Ergonomía aplicada a la movilización de pacientes en un servicio de hospitalización mediante el método MAPO. Revista Enfermería del Trabajo, 6(2), 43-50. https://dialnet.unirioja.es/servlet/articulo?codigo $=5476839$

Alcazar-Belchí, M. (2015). Prevención de riesgos laborales en la movilización de pacientes en una planta de hospitalización [tesis de maestría en Prevención en Riesgos Laborales, Universidad Miguel Hernández]. Repositorio RediUMH. http://hdl.handle.net/11000/2154

Álvarez-Casado, E., Hernández-Soto, A.C., \& Rayo-García, V. (2010). El riesgo asociado a la movilización de pacientes. Wolters Kluwer España S.A. https://upcommons.upc.edu/handle/2117/12223

Battevi, N., Menoni, O., Grazia-Ricci, M., \& Cairoli, S. (2006). MAPO index for risk assessment of patient manual handling in hospital wards: a validation study. Ergonomics, 49(7), 671-687. https://doi.org/10.1080/00140130600581041

Bernal-Osorio, M., Fernández-Carrera, C., \& Uribe-López, J.F. (2018). Aplicación del método "MAPO" para evaluar el riesgo biomecánico por movilización de pacientes en auxiliares de enfermería del hospital General San Isidro de la ciudad de Manizales [trabajo de grado en el programa de Especialización en Seguridad y Salud en el Trabajo, Universidad Católica de Manizales]. Repositorio UCM. http://repositorio.ucm.edu.co:8080/jspui/handle/10839/1974

Chen, J. \& Yang, L. (2006). Maximum acceptable weight of lift for manual lifting tasks. Chinese Journal of Industrial Hygiene and Occupational Diseases 24(4), 194-197. https://pubmed.ncbi.nlm.nih.gov/16701027/

Díaz, P., Gutiérrez, E., \& Meneses, A. (2017). Papel de la ergonomía hospitalaria frente al manejo del paciente adulto por personal de auxiliar de enfermería. Revista QUID, (28), 37-45. https://revistas.proeditio.com/iush/quid/article/view/1664

Jäger, M., Jordan, C., Theilmaier, A., Luttmann, A. \& The Dolly Group (2010). Lumbar load quantification and overload - risk prevention for manual patient handling - The Dortmund Approach. In Proc. 8th Int Conf Occup Risk Prevention ORP 2010. [CD-rom.] Valencia, Spain.

Jorgensen, M.J., Davis, K.G., Kiring, B.C., Lewis, K.E., \& Marras, W.S. (1999). Significance of biomechanical and physiological variables during the determination of maximum acceptable weight of lift. Ergonomics, 42(9), 1216-1232. https://doi.org/10.1080/001401399185090

Karhula, K., Härmä, M., Sallinen, M., Hublin, C., Virkkala, J., Kivimäki, M., Vahtera, J., \& Puttonen, S. (2013). Association of job strain with working hours, shift-dependent perceived workload, sleepiness and recovery. Ergonomics, 56(11), 1640-1651. https://doi.org/10.1080/00140139.2013.837514

Karhula, K., Rönnholm, T. \& Sjögren T. (2009). A method for evaluating the load of -patient 
transfers. Occupational Safety and Health Administration. https://converis.jyu.fi/converis/portal/detail/Publication/19291864

Knibbe, J. J., \& Friele, R. D. (1999). The use of logs to assess exposure to manual handling of patients, illustrated in an intervention study in home care nursing. International Journal of Industrial Ergonomics, 24(4), 445-454. https://doi.org/10.1016/s0169-8141(99)ooo10-4

Knibbe, J.J. \& Knibbe, N.E. (2012). An international validation study of the care thermometer: a tool supporting the quality of ergonomic policies in health care. Work, 41, 5639- 5641. DOI: https://doi.org/10.3233/WOR-2012-0903-5639

Knibbe, J.J., Knibbe, N.E. \& Waaijer, E. (2012). Flying through the hospital: Efficiency and safety of an ergonomic solution. Work, 41, 5642-5643. https://doi.org/10.3233/WOR-2012-09045642

Malo, M.A., Cueto, B., García, C. \& Pérez, J.I. (2012). La medición del absentismo: Estimaciones desde la perspectiva de las empresas y de las vidas laborales. Ministerio de Empleo y Seguridad Social

http://www.segsocial.es/prdioo/groups/public/documents/binario/174212.pdf

Montalvo-Prieto, A., Cortés-Múnera, Y., \& Rojas-López, M. (2015). Riesgo ergonómico asociado a sintomatología musculo esquelética en personal de enfermería. Revista Hacia la Promoción de la Salud, 20(2), 132-146. https://doi.org/10.17151/hpsal.2015.20.2.11

Morales-Perrazo, L.A., Aldás-Salazar, D.S., Collantes-Vaca, M.S., \& Freire, J.V. (2017). Ergonomía del trabajo de enfermeras en el manejo manual de pacientes con metodología REBA y MAPO. Revista digital de Medio Ambiente "Ojeando la agenda", 1(48), 1-17.

Noriega-Elío, M., Barrón-Soto, A., Sierra- Martínez, O., Méndez-Ramírez, I., Pulido-Navarro, M. \& Cruz-Flores, C. (2005). La polémica sobre las lumbalgias y su relación con el trabajo: Estudio retrospectivo en trabajadores con invalidez. Cad. Saúde Pública, 21(3), 887-897. http://dx.doi.org/10.1590/So102-311X2005000300023

Puello-Mercado, H. \& Medina, A. (2017). Nivel de riesgo biomecánico por manipulación manual de pacientes adultos en el área de hospitalización de una institución de salud de alta complejidad en Soledad, 2016-1 [tesis de maestría en Seguridad y Salud en el Trabajo, Universidad del Norte $\quad-\quad$ Colombia]. Repositorio UniLibre. https://repository.unilibre.edu.co/handle/10901/10684

Robla-Santos D. (2015). El papel de la ergonomía en la prevención y evaluación de la carga física en un centro hospitalario: Evaluación de las tareas de movilización de pacientes mediante el método MAPO Index [tesis doctoral en nuevas Tendencias y aplicaciones en el ámbito asistencial, Universidad de La Coruña-España]. Repositorio Universidad de Coruña. https://ruc.udc.es/dspace/handle/2183/16452

Robla-Santos, D., Hernández-Soto, A., Riveiro, S., \& Rodríguez, B. (2010). Índice MAPO para la evaluación del riesgo por manipulación manual de pacientes en quirófanos. VII Congreso Internacional ORP. Valencia.

Salazar-Montes, A.M., Murcia-Paredes, L.M., \& Solano-Pérez, J.A. (2016). Evaluación e intervención de la sobrecarga del cuidador informal de adultos mayores dependientes: Revisión de artículos publicados entre 1997 - 2014. Arch Med (Manizales), 16(1):144-54. 
https://www.redalyc.org/jatsRepo/2738/273846452015/index.html

Sánchez, R.T., Molina, E., \& Gómez-Ortega, O. (2016). Intervenciones de enfermería para disminuir la sobrecarga en cuidadores: Un estudio piloto. Revista Cuidarte, 7(1), 1171-84. https://doi.org/10.15649/cuidarte.v7i1.251

Tamminen-Peter, L., Fagerström, V. \& Moilanen, A. (2008). Comparison of risk assessment tools of patient handling. Finish Institute of Occupational Health. https://www.researchgate.net/publication/270785861 Comparison of risk assessment $t$ ools of patient handling

Uvida, M. (2016). Equipo biomecánico para la movilización de pacientes sin autonomía en el personal de enfermería del hospital Andino Alternativo de Chimborazo [tesis de maestría en Seguridad Industrial, prevención de riesgos y salud ocupacional, Universidad Nacional de Chimborazo - $\quad$ Ecuador]. Repositorio Digital UNACH. http://dspace.unach.edu.ec/handle/51000/3153 